\title{
Primary osteosarcoma in elderly patients: A report of three cases
}

\author{
QIANG XU*, TIAN GAO* ${ }^{*}$ BIN ZHANG, JIN ZENG ${ }^{* *}$ and MIN DAI** \\ Department of Orthopedics, The First Affiliated Hospital of Nanchang University, \\ Artificial Joints Engineering and Technology Research Center of Jiangxi Province, Nanchang, Jiangxi 330006, P.R. China
}

Received September 8, 2018; Accepted March 7, 2019

DOI: $10.3892 / \mathrm{ol} .2019 .10446$

\begin{abstract}
Osteosarcoma is the most common type of primary malignant bone tumor in children and young adults. However, primary osteosarcoma in elderly patients is rare. The present study reports 3 cases of advanced osteosarcoma in elderly patients. The pathological findings in all 3 cases confirmed the diagnosis of primary osteosarcoma. Notably, each patient received different treatment options. Chemoradiotherapy was recommended in case 1 due to the age of the patient. However, the patient requested to be discharged and was lost to follow-up. Conversely, in case 2, the 62-year-old female patient underwent systemic chemotherapy, but no surgical treatment, and in case 3, the 51-year-old male patient underwent complete tumor resection and received systemic chemotherapy for late tumor recurrence. Early diagnosis of osteosarcoma in elderly patients is difficult, and misdiagnosis or a missed diagnosis is common. In clinical practice, bone tumors in elderly patients should be investigated carefully. Imaging examinations are essential for diagnosis, and biopsy is required for confirmation. However, the efficacy of chemotherapy for elderly patients with primary osteosarcoma remains uncertain. Collectively, due to the small number of reports of osteosarcoma in the elderly population, the 3 cases in the present study raise awareness of this rare condition.
\end{abstract}

\section{Introduction}

Osteosarcoma is a primary malignant tumor of the skeleton characterized by direct formation of bone or bone-like tissue by tumor cells $(1,2)$. Osteosarcoma predominantly occurs in adolescents and is the most common type of primary bone

Correspondence to: Dr Jin Zeng or Professor Min Dai, Department of Orthopedics, The First Affiliated Hospital of Nanchang University, Artificial Joints Engineering and Technology Research Center of Jiangxi Province, 17 Yong Wai Zheng Street, Nanchang, Jiangxi 330006, P.R. China

E-mail: 774735825@qq.com

E-mail: daimin@medmail.com.cn

\section{${ }^{*, * *}$ Contributed equally}

Key words: osteosarcoma, elderly patients, primary malignant bone tumor, surgery, chemoradiotherapy, imaging, pathology tumor, with an annual incidence of 1-3 cases per million individuals, accounting for $\sim 0.2 \%$ of malignant tumors and $\sim 15 \%$ of primary bone tumors (3-6). The most common sites for tumor development are the long bones, typically the distal femur or proximal humerus; manifestation at other sites is rare $(7,8)$. Hematogenous metastasis occurs early and has a high incidence, rapid progression, high mortality and is difficult to treat $(9,10)$. Therefore, in recent years, the study of osteosarcoma has become an important issue in the medical community (11).

The age of onset of osteosarcoma has two peaks. The first peak is in children and adolescents aged 10-20 years, accounting for $60 \%$ of all cases of osteosarcoma; the second peak is in adults aged $>40$ years, accounting for $13 \%$ of all osteosarcomas (12). There are a number of studies on osteosarcoma in children and adolescents. When osteosarcoma occurs in patients aged $>40$ years, it is usually considered to be secondary to Paget's disease of bone or irradiated bone lesions (13); thus, primary osteosarcoma in older adults is rare. Previous studies have concluded that patients with osteosarcoma have a worse prognosis with increasing age $(14,15)$. Since 2007, 198 primary osteosarcomas have been diagnosed and treated in the Department of Orthopedics, The First Affiliated Hospital of Nanchang University, (Nanchang, Jiangxi, China). The number of patients aged $>50$ years is $<8$, including the 3 elderly patients described in the present report. Currently, there are no reports of a patient aged $>79$ years with a diagnosis of primary osteosarcoma. In the present study, the clinical characteristics, imaging and pathological findings, diagnosis and treatment of 3 cases of adult and elderly patients with primary osteosarcoma are discussed. The First Affiliated Hospital of Nanchang University ethical review committee approved the present study, and written informed consent was obtained from all patients.

\section{Case report}

Case 1. In December 2017, a 79-year-old Chinese female retired worker (height, $155 \mathrm{~cm}$; weight, $45 \mathrm{~kg}$ ) visited the Department of Orthopedics, The First Affiliated Hospital of Nanchang University outpatient clinic with a 1-month history of pain in the left groin that was relieved following rest. The pain did not bother the patient at that time, but it had increased in the previous week. The patient had no history of injuries or illnesses, and no relevant family history. Physical examination revealed that the skin around the left inguinal region was 
tender to the touch, but displayed no redness, varicose veins or hyperpigmentation. The left lower extremity motion and sensation were within normal limits. There was no obvious abnormality in the other extremities.

During laboratory examination, the following levels were detected: Erythrocyte sedimentation rate, $85 \mathrm{~mm} / \mathrm{h}$; C-reactive protein level, $25.20 \mathrm{mg} / \mathrm{ml}$; serum alkaline phosphatase level, 214 units/l; carbohydrate antigen 724 level, 24.35 units $/ \mathrm{ml}$; and squamous cell carcinoma-associated antigen level, $3.19 \mathrm{ng} / \mathrm{ml}$; all of which were higher than the normal ranges $(0-20 \mathrm{~mm} / \mathrm{h}$, 0-8 mg/ml, 50-135 units/1, 0-6.9 units $/ \mathrm{ml}$, and 0-2.5 ng/ml, respectively). Radiography revealed bony changes of the upper left suprapubic branch, ischium and acetabulum (Fig. 1A). CT demonstrated irregular bone destruction of the left acetabulum and ischium, adjacent soft tissue swelling, and a visible shadow. The soft tissue mass measured approximately $6.9 \times 4.0 \mathrm{~cm}$ and had an ill-defined boundary. There were plaque-like, cloud-shaped, needle-like, high-density shadows in the left hip joint, but no obvious abnormalities in the right hip joint (Fig. 1B). A whole body bone scan revealed increased bone metabolism in the left acetabulum, ischium, and femoral head, which was attributed to a malignant bone lesion. Increased bone metabolism in the fourth and fifth ribs and T8 vertebra was also observed (Fig. 1C).

Using hematoxylin and eosin staining, the left ilium bone tumor revealed heterogeneous cells diffusely distributed between trabecular bone. In a fragment of fibrous tissue and muscle tissue, oval-shaped tumor cells with large nuclei were distributed in patches and karyolysis was present. The tumor cells had an invasive growth pattern (Fig. 2A and B). Taking into account the age and poor physical status of the patient, chemoradiotherapy was recommended. However, the patient and the patients' family did not wish to continue treatment and requested to be discharged. The patient was discharged 2 weeks after admission and was lost to follow-up.

Case 2.In November 2016, a 62-year-old Chinese female farmer (height, $157 \mathrm{~cm}$; weight, $48 \mathrm{~kg}$ ) presented with a left femoral mass of uncertain etiology and pain in the left lower extremity had been experienced for 2 months. The patient was admitted at a The First People's Hospital of Xiaoshan City (Xiaoshan, China), and a diagnosis of osteomyelitis was considered. The patient was admitted to the Department of Orthopedics, The First Affiliated Hospital of Nanchang University for further treatment. The patient had no previous medical history.

The patient had no history of injury, past medical illnesses or family history that were associated with disease. Physical examination revealed a $10 \times 5 \times 5 \mathrm{~cm}$ hard mass of the distal left femur; there was no obvious redness or ulceration in the adjacent skin. There was also no significant limitation in the activity level of the patient and no neurovascular defects in the lower limbs.

Routine laboratory testing revealed a neuron-specific enolase level of 24.26 units $/ \mathrm{ml}$ (normal range, $0-16.3$ units $/ \mathrm{ml}$ ) and an erythrocyte sedimentation rate of $26 \mathrm{~mm} / \mathrm{h}$ (normal range, $0-20 \mathrm{~mm} / \mathrm{h}$ ). Radiography performed at the local hospital revealed a low-density shadow at the distal end of the left femur. CT demonstrated a soft tissue mass shadow and a bony mass shadow attached to the surface of the bone at the distal left femur (Fig. 1D and E). Enhanced MRI revealed an area of irregular bone destruction in the distal femur, and an abnormal signal shadow was observed (Fig. 1G). A whole body bone scan revealed a cluster of lesions at the distal end of the left femur (Fig. 1F).

Using hematoxylin and eosin staining, the left femur bone biopsy revealed proliferating spindle cells in bundles or braids. The tumor cells had lightly stained cytoplasm, oval nuclei and no nuclear division. A red-stained matrix was visible, peripheral osteoblasts were adhered to adjacent structures and areas of calcification were observed (Fig. 2C and D). The patient received two cycles of local arterial perfusion as follows: Cisplatin (DDP) was administered at a dose of $120 \mathrm{mg}$ on day 1 , and the regimen was repeated every 14 days. After 3 months, the patient was given six cycles of DDP (120 mg) once daily for 1 day as systemic intravenous chemotherapy; $20 \mathrm{mg}$ doxorubicin once daily for 3 days, after 2 weeks of $2 \mathrm{~g}$ isophosphate for 5 days. After 2 weeks, the cycle was repeated. At the follow-up 2.5 years after surgery, there was no significant pain in the left lower extremity and no evidence of local or distant recurrence following chemotherapy.

Case 3. In March 2013, a 51-year-old Chinese male teacher (height, $168 \mathrm{~cm}$; weight, $56 \mathrm{~kg}$ ) sustained a left femoral neck fracture due to a fall and underwent left total hip arthroplasty in Xinjian County People's Hospital (Nanchang, China). The postoperative recovery of the patient was unremarkable. However, at 2 years after surgery, the patient developed left hip pain of unclear etiology with limited mobility. Due to the marked increase in pain and limited mobility, the patient presented to the Department of Orthopedics, The First Affiliated Hospital of Nanchang University for further treatment and was admitted in October 2015. The patient had no history of allergies or related family history. Postoperative physical examination revealed a $15-\mathrm{cm}$ healing scar on the left hip, tenderness of the left femoral trochanter, elevated local skin temperature, no redness of the skin, limited left hip joint activity and normal left knee joint activity. There were no obvious abnormalities of the muscles and muscle tone of the lower limbs. There was also no significant recent change in the weight of the patient.

Routine laboratory testing revealed a serum alkaline phosphatase level of 785 units/l (normal range, 50-135 units/l) and a C-reactive protein level of $10.8 \mathrm{mg} / \mathrm{ml}$ (normal range, $0-8 \mathrm{mg} / \mathrm{l}$ ), while the erythrocyte sedimentation rate and tumor markers (including $\alpha$-fetoprotein, carcinoembryonic antigen, carbohydrate antigen 12-5, carbohydrate antigen 15-3 and carbohydrate antigen 19-9) were within the normal ranges. Radiography observed the artificial hip joint replacement on the left side, as well as a soft tissue mass shadow visible in the upper left femur, which suggested malignancy (Fig. 1H). A whole body bone scan revealed concentrated foci in the left upper femur and the left knee joint, which were suggestive of ipsilateral metastasis (Fig. 1I). The patient was initially diagnosed with bone metastasis.

Following routine preoperative assessment, continuous spinal dural anesthesia was used, and the patient was placed in the right lateral position. Following the extension of the original surgical incision, the skin, subcutaneous tissue, and superficial fascia were dissected layer by layer; subsequently, the fascia was opened, and the muscle fibers of the gluteus maximus were dissected to reveal the proximal femur. The 

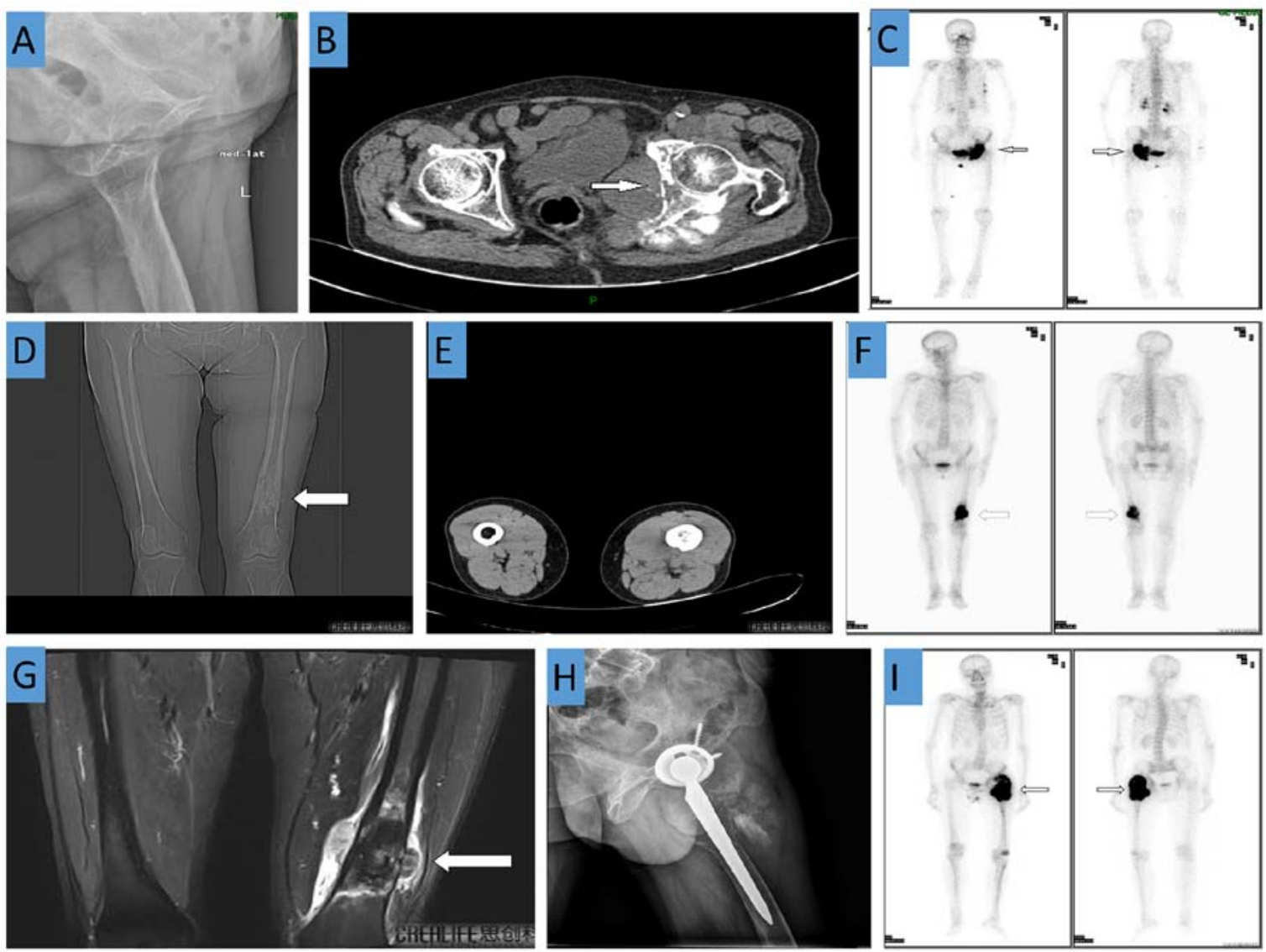

Figure 1. Case 1: (A) X-ray revealed bony changes in the upper left suprapubic branch, ischium and acetabulum; (B) CT demonstrated irregular bone destruction of the left acetabulum and ischium and adjacent soft tissue swelling; (C) A whole body bone scan revealed increased bone metabolism in the left acetabulum, ischium, femoral head, left fourth and fifth ribs and T8 vertebra. Case 2: CT demonstrates a soft tissue mass shadow and a bony mass shadow attached to the surface of the bone at the distal end of the left femur. (D) Coronal view. (E) Transverse view. (F) Increased bone metabolism at the distal end of the left femur. (G) MRI of the distal femur shows irregular bone destruction with an abnormal signal shadow. Case 3: (H) X-ray shows that after left artificial hip joint replacement, a soft tissue mass shadow is visible in the upper left femur; (I) A whole body bone scan revealed increased bone metabolism in the left upper femur.

original femoral prosthesis was removed, and upper femoral bone destruction was visualized. In the surgical field of view, the tumor appeared similar to the flesh of a fish and invaded the surrounding soft tissue, with ill-defined margins. Moreover, intraoperative cryosectioning revealed malignant tumor cells. Subsequently, granulation tissue within the medullary cavity, the surrounding tumor tissue, and the surrounding affected muscle tissue were completely removed. Subsequently, a customized tumor prosthesis was implanted into the medullary canal and fixed with bone cement. As the original acetabular cup was tilted backward, polyethylene was added to the trailing edge to prevent dislocation. Following resetting of the cup, the hip joint was examined for completeness and satisfactory mobility. Finally, the wound was soaked in distilled water, and was repeatedly washed with hydrogen peroxide and physiological saline. A drainage tube was placed under the muscle layer, the fascia was sutured, and the incision was closed layer by layer.

Histological examination [with hematoxylin and eosin (HE) staining] of the tumor from the left proximal femur revealed irregular, bone-like tissues arranged in a trabecular or mesh shape. The tumor cells revealed large deeply stained nuclei, abundant red-stained or translucent cytoplasm, and a large number of giant tumor cells. In some areas, fragments of bone tissue and immature cartilage tissue were visualized
(Fig. 2E and F). Based on the medical history of the patient, the imaging and laboratory findings, and the pathological results, the final diagnosis of primary osteosarcoma of the proximal left femur was established.

Postoperatively, adjuvant chemotherapy was recommended. However, the patient and the patient's family declined treatment and requested discharge, but agreed to follow-up. The patient revealed no signs of recurrence within 3 months after surgery and no signs of distant metastasis. However, 6 months after surgery, the patient revealed evidence of tumor recurrence on the postoperative follow-up imaging examination (Fig. 3). The patient received a combination of gemcitabine/docetaxel as follows: $900 \mathrm{mg} / \mathrm{m}^{2}$ gemcitabine on days 1 and 8 , and $120 \mathrm{mg} / \mathrm{m}^{2}$ docetaxel on day 8 . The regimen was repeated every 3 weeks, and although the patient continued to be treated for 6 months, the left hip pain of this patient did not improve following chemotherapy, and the activity was limited.

\section{Discussion}

The majority of studies on the diagnosis and treatment of osteosarcoma have focused on adolescents. Osteosarcoma at other ages is rarely discussed, with the exception of osteosarcoma secondary to Paget's disease ( $0.2 \%$ of osteosarcomas) (16) or following radiotherapy (17). In the present study, the records 

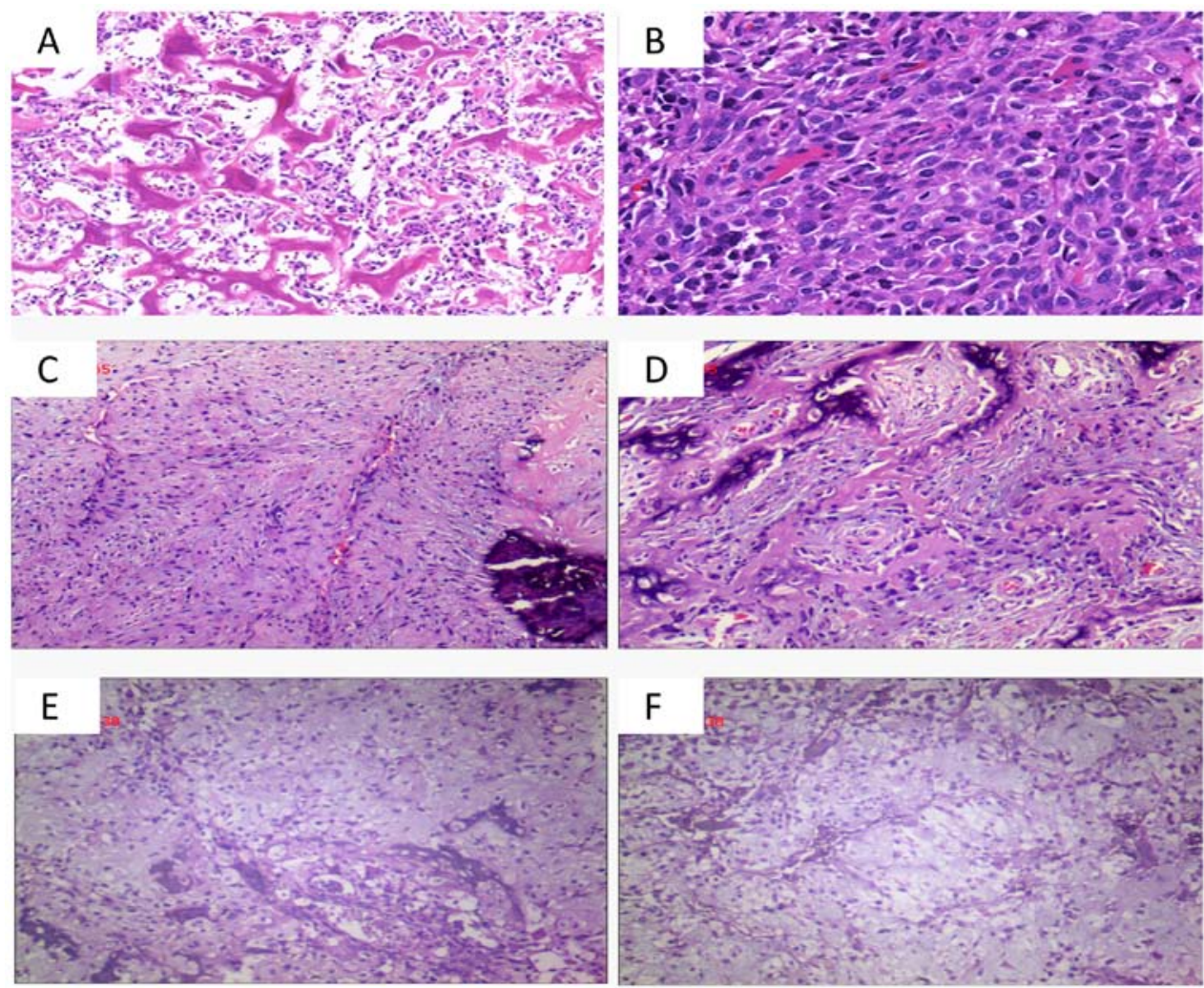

Figure 2. Post-operative histological observations. (A and B) Case 1, diffuse distribution of tumor cells between the trabecular bone and tumor cells in fibrous tissue and muscle tissue are distributed in patches and reveal infiltrating growth (A, HE staining; magnification, x100; B, HE staining; magnification, x200). (C and D) Case 2, spindle cells in the tissue proliferating in bundles or weave patterns, adherent peripheral osteoblasts, and calcification were observed (HE staining; magnification, x100). (E and F) Case 3, a large number of irregular bone-like structures are arranged in trabecular or reticulated patterns, and damaged bone tissue and immature cartilage (HE staining; magnification, $x 100)$. HE, hematoxylin and eosin.
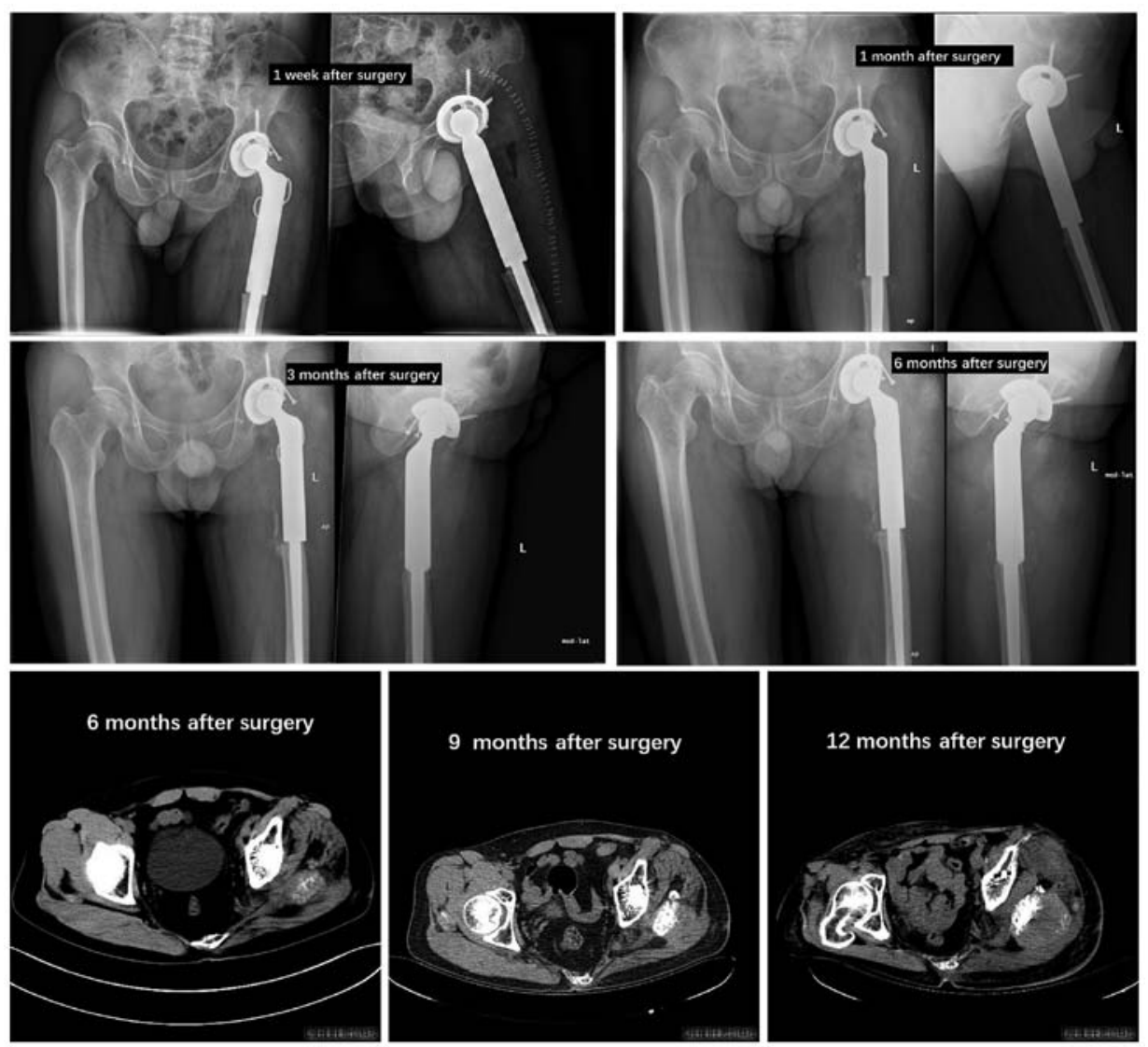

Figure 3. X-ray or CT imaging data at patient follow-up in case 3. 
Table I. Differential diagnosis of osteosarcoma in middle-aged and elderly patients.

Differential diagnosis

Details

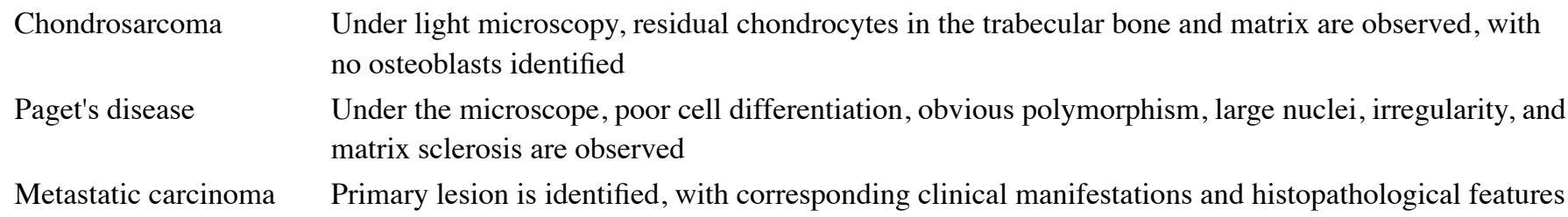

of 3 patients with primary osteosarcoma $>50$ years of age and treated in the Department of Orthopedics, The First Affiliated Hospital of Nanchang University were reviewed, in order to demonstrate the clinical features and prognostic factors of this disease.

The diagnosis of osteosarcoma is based on the combination of clinical, radiography and pathological results. Pathological confirmation of the formation of osteoid tissue and/or bone by tumor cells is key to the diagnosis. Various unusual histological subtypes were more common in these 3 older patients compared with that in younger patients, and fibroblasts and chondrocyte subtypes were observed in addition to the conventional subtypes of osteoblasts. However, early diagnosis of osteosarcoma in older patients is difficult, and misdiagnosis or a missed diagnosis is common (15). This may be associated with the following factors: i) The incidence of osteosarcoma in elderly individuals is extremely rare $(18,19)$; ii) osteosarcoma in elderly individuals can be confused with osteoarthropathy (15); iii) following the discovery of lesions, clinicians tend to focus on bone metastases and other primary tumors that occur more commonly in elderly individuals (20). For example, chondrosarcoma occurs predominantly in the elderly (21). In some cases, focal ossification can be visualized under the microscope; this feature is similar to that observed for osteosarcoma (2). However, intraosseous ossification of chondrosarcoma appears as trabecular bone in a background of cartilage (22). In addition, residual chondrocytes are observed in trabecular bone, as well as around the stroma (23).

A key finding detected by light microscopy is the absence of tumor osteogenesis in chondrosarcoma (24). For metastatic cancer, the primary lesion should be identified, and the corresponding clinical manifestations and histopathological features should be linked with the bone tumor. Although malignant fibrous histiocytoma is more common in the soft tissues, it can also occur in bone in elderly populations. In these cases, it is easy to distinguish osteosarcoma from malignant fibrous histiocytoma based on imaging and pathological findings. Previous reports have focused on osteosarcoma in children and adolescents; however, osteosarcoma is often secondary to Paget's disease in elderly patients $(12,25)$. In Western populations, secondary primary osteosarcoma accounts for a large proportion of elderly patients with osteosarcoma, usually due to Paget's disease, benign bone disease or radiation complications $(26,27)$. No patients with primary osteosarcoma had Paget's disease in the present study. Histologically, poor cell differentiation, obvious polymorphism, large nuclei, irregularity and matrix sclerosis were observed. However, in these
3 patients, there was a large volume of immature or mature bone-like stroma between the tumor cells, which is very different from the appearance of osteosarcoma secondary to Paget's disease. As the majority of patients with Paget's disease have no obvious symptoms, when osteosarcoma occurs in older patients, the diagnosis of primary osteosarcoma can be made following the exclusion of osteosarcoma secondary to Paget's disease. Table I summarizes the differential diagnosis of osteosarcoma in elderly patients.

In the 1970s, the standard treatment for osteosarcoma was amputation, but the 5-year survival rate was $<20 \%$ (28). In the 1980 s, due to advances in surgical techniques, the development of effective chemotherapy, and improved preoperative and postoperative chemoradiotherapy procedures, limb-salvage surgery gradually replaced traditional amputation (29). The key to the success of limb salvage surgery lies in the choice of the surgical method and removal of the tumor according to the most appropriate surgical boundary (30). Osteosarcoma should be completely removed to avoid local recurrence and distant metastasis, with the resection plane $5-7 \mathrm{~cm}$ from the end of the lesion. If the lesion is not completely removed during surgery, the local recurrence rate can be as high as $25 \%$ (31). Rosen et al (32). advocated neoadjuvant chemotherapy for osteosarcoma. The 5-year survival rate in patients has increased from 20 to $50-70 \%$. In addition, up to $60-80 \%$ of patients can obtain limb retention, which has become the standard treatment for osteosarcoma currently accepted by the majority of scholars; namely 'neoadjuvant chemotherapy + surgery (limb salvage or amputation) + adjuvant chemotherapy'. Certain reports have stated that chemotherapy is beneficial in elderly patients with primary osteosarcoma $(33,34)$. However, in 1986, Huvos (35) reported that among 117 cases of osteosarcoma in bone and soft tissue that occurred in patients aged $>60$ years, $44 \%$ had primary osteosarcoma; the 5-year survival rate following treatment was $18 \%$, and the 10 -year survival rate was $7 \%$, suggesting that patients had a poor prognosis. In the present study, case 2 received systematic chemotherapy and achieved a good therapeutic effect. However, case 3 also received chemotherapy but did not achieve satisfactory results. Therefore, regarding the effect of chemotherapy on osteosarcoma in elderly patients, the number of studies is insufficient to show whether chemotherapy is effective.

In conclusion, one of the main limitations of the present study is its insufficient number of cases. In addition, the 3 patients had different treatments therefore no comparisons could be determined. Osteosarcoma in elderly patients is a possible cause of pain and should not be ignored. Given its 
rarity, primary osteosarcoma should be carefully evaluated. We hypothesize that with increased awareness, it is possible to reach a correct diagnosis in advance to avoid over-treatment. Finally, osteosarcoma in elderly patients is rare; in order to improve the understanding of this condition, more case studies are required. Plastic surgeons and radiologists should consider the possibility of osteosarcoma diagnosis in this age group.

\section{Acknowledgements}

Not applicable.

\section{Funding}

The present study was supported by Health and Family Planning Commission of Jiangxi Province on Traditional Chinese Medicine (grant no. 2016A073).

\section{Availability of data and materials}

All data generated or analyzed during the present study are included in this published article.

\section{Authors' contributions}

$\mathrm{MD}$, and $\mathrm{JZ}$ performed the surgery and managed the patients postoperatively. QX and TG contributed to the concept and design of the study and to the acquisition, analysis or interpretation of working data. BZ analyzed the patient's data. All authors read and approved the final manuscript.

\section{Ethics approval and consent to participate}

The study was approved by the Ethical Institutional Review Board of the First Affiliated Hospital of Nanchang University, and written informed consent was obtained from all study participants.

\section{Patient consent for publication}

The consent for publication of the manuscript and the related images from the patients and/or their relatives was obtained by the First Affiliated Hospital of Nanchang University.

\section{Competing interests}

The authors declare that they have no competing interests.

\section{References}

1. Kansara M, Teng MW, Smyth MJ and Thomas DM: Translational biology of osteosarcoma. Nat Rev Cancer 14: 722-735, 2014.

2. Poletajew S, Fus L and Wasiutynski A: Current concepts on pathogenesis and biology of metastatic osteosarcoma tumors. Ortop Traumatol Rehabil 13: 537-545, 2011 (In English, Polish).

3. Centrella M, Horowitz MC, Wozney JM and McCarthy TL: Transforming growth factor-beta gene family members and bone. Endocr Rev 15: 27-39, 1994.

4. Bismar H, Klopinger T, Schuster EM, Balbach S, Diel I, Ziegler R and Pfeilschifter J: Transforming growth factor beta (TGF-beta) levels in the conditioncd media of human bone cclls relationship to donor age bone volvme and cconcentration of TGF-beta in human bone matrix in vivo. Bone 24: 565-569, 1999.
5. Savage SA and Mirabello L: Using epidemiology and genomics to understand osteosarcoma etiology. Sarcoma 2011: 548151, 2011.

6. Meyers PA and Gorlick R: Osteosarcoma. Pediatr Clin North Am 44: 973-989, 1997.

7. Damron TA, Ward WG and Stewart A: Osteosarcoma, chondrosarcoma, and Ewing's sarcoma: National cancer data base report. Clin Orthop Relat Res 459: 40-47, 2007.

8. Anil S, Krishnan AP and Rajendran R: Osteosarcoma of the mandible masquerading as a dental abscess: Report of a case. Case Rep Dent 2012: 635062, 2012.

9. Tsuchiya H, Kanazawa Y, Abdel-Wanis ME, Asada N, Abe S, Isu K, Sugita T and Tomita K: Effect of timing of pulmonary metastases identification on prognosis of patients with osteosarcoma: The japanese musculoskeletal oncology group study. J Clin Oncol 20: 3470-3477, 2002.

10. Williams RF, Fernandez-Pineda I and Gosain A: Pediatric sarcomas. Surg Clin North Am 96: 1107-1125, 2016.

11. Haduong JH, Martin AA, Skapek SX and Mascarenhas L: Sarcomas. Pediatr Clin North Am 62: 179-200, 2015.

12. Duffaud F, Digue L, Baciuchka-Palmaro M, Volot F, Perles-Daniel C, Garbe L and Favre R: Osteosarcomas of flat bones in adolescents and adults. Cancer 88: 324-332, 2000.

13. Ek ET, Ojaimi J, Kitagawa Y and Choong PF: Outcome of patients with osteosarcoma over 40 years of age: Is angiogenesis a marker of survival? Int Semin Surg Oncol 3: 7, 2006.

14. Durnali A, Alkis N, Cangur S, Yukruk FA, Inal A, Tokluoglu S, Seker MM, Bal O, Akman T, Inanc M, et al: Prognostic factors for teenage and adult patients with high-grade osteosarcoma: An analysis of 240 patients. Med Oncol 30: 624, 2013.

15. Sadoghi P, Leithner A, Clar H, Glehr M, Wibmer C, Bodo K, Quehenberger $\mathrm{F}$ and Windhager R: The threat of misdiagnosis of primary osteosarcoma over the age of 60: A series of seven cases and review of the literature. Arch Orthop Trauma Surg 130: 1251-1256, 2010.

16. Wick MR, Siegal GP, Unni KK, McLeod RA and Greditzer HG III: Sarcomas of bone complicating osteitis deformans (Paget's disease): Fifty years' experience. Am J Surg Pathol 5: 47-59, 1981.

17. Frassica FJ, Frassica DA, Wold LE, Beabout JW and Sim FH: Postradiation sarcoma of bone. Orthopedics 16: 105-109, 1993.

18. Ogura K, Higashi T and Kawai A: Statistics of bone sarcoma in Japan: Report from the bone and soft tissue tumor registry in Japan. J Orthop Sci 22: 133-143, 2017.

19. Ishikawa $Y$, Tsukuma $H$ and Miller RW: Low rates of Paget's disease of bone and osteosarcoma in elderly Japanese. Lancet 347: $1559,1996$.

20. Jeon DG, Lee SY, Cho WH, Song WS and Park JH: Primary osteosarcoma in patients older than 40 years of age. J Korean Med Sci 21: 715-718, 2006.

21. Nie Z, Lu Q and Peng H: Prognostic factors for patients with chondrosarcoma: A survival analysis based on the Surveillance, Epidemiology, and End Results (SEER) database (1973-2012). J Bone Oncol 13: 55-61, 2018.

22. Sergi $\mathrm{C}$ and Zwerschke W: Osteogenic sarcoma (osteosarcoma) in the elderly: Tumor delineation and predisposing conditions. Exp Gerontol 43: 1039-1043, 2008.

23. Lex JR, Evans S, Stevenson JD, Parry M, Jeys LM and Grimer RJ: Dedifferentiated chondrosarcoma of the pelvis: Clinical outcomes and current treatment. Clin Sarcoma Res 8: 23, 2018.

24. Gumay S: Pathological diagnosis of bone sarcoma. Gan To Kagaku Ryoho 27 (Suppl 2): S420-S426, 2000.

25. Bielack SS, Kempf-Bielack B, Heise U, Schwenzer D and Winkler K: Combined modality treatment for osteosarcoma occurring as a second malignant disease. Cooperative German-Austrian-Swiss Osteosarcoma Study Group. J Clin Oncol 17: 1164, 1999.

26. Grimer RJ, Cannon SR, Taminiau AM, Bielack S, Kempf-Bielack B, Windhager R, Dominkus M, Saeter G, Bauer H, Meller I, et al: Osteosarcoma over the age of forty. Eur J Cancer 39: 157-163, 2003

27. deSantos LA, Rosengren JE, Wooten WB and Murray JA: Osteogenic sarcoma after the age of 50: A radiographic evaluation. AJR Am J Roentgenol 131: 481-484, 1978.

28. Ferrari S and Palmerini E: Adjuvant and neoadjuvant combination chemotherapy for osteogenic sarcoma. Curr Opin Oncol 19: 341-346, 2007.

29. Wittig JC, Bickels J, Priebat D, Jelinek J, Kellar-Graney K, Shmookler B and Malawer MM: Osteosarcoma: A multidisciplinary approach to diagnosis and treatment. Am Fam Physician 65: 1123-1132, 2002. 
30. Agarwal M, Anchan C, Shah M, Puri A and Pai S: Limb salvage surgery for osteosarcoma: Effective low-cost treatment. Clin Orthop Relat Res 459: 82-91, 2007.

31. Kim HJ, Chalmers PN and Morris CD: Pediatric osteogenic sarcoma. Curr Opin Pediatr 22: 61, 2010.

32. Rosen G, Caparros B, Huvos AG, Kosloff C, Nirenberg A, Cacavio A, Marcove RC, Lane JM, Mehta B and Urban C: Preoperative chemotherapy for osteogenic sarcoma: Selection of postoperative adjuvant chemotherapy based on the response of the primary tumor to preoperative chemotherapy. Cancer 49: 1221-1230, 1982

33. Bacci G, Ferrari S, Donati D, Longhi A, Bertoni F, Di Fiore M, Comandone A, Cesari M and Campanacci M: Neoadjuvant chemotherapy for osteosarcoma of the extremity in patients in the fourth and fifth decade of life. Oncol Rep 5: 1259-1263, 1998
34. Manoso MW, Healey JH, Boland PJ, Athanasian EA, Maki RG, Huvos AG and Morris CD: De novo osteogenic sarcoma in patients older than forty: Benefit of multimodality therapy. Clin Orthop Relat Res 438: 110-115, 2005.

35. Huvos AG: Osteogenic sarcoma of bones and soft tissues in older persons. A clinicopathologic analysis of 117 patients older than 60 years. Cancer 57: 1442-1449, 1986.

This work is licensed under a Creative Commons Attribution-NonCommercial-NoDerivatives 4.0 International (CC BY-NC-ND 4.0) License. 